Personality, particularly conscientiousness and agreeableness appears to impact the development of physician burnout. Strategies that modulate the relationship between personality and burnout may be beneficial for optimal health care delivery. Further research is needed to identify appropriate short and longterm strategies to ensure physician wellbeing and optimal delivery of patient care.

\section{Mental health training for correctional officers: a systematic review}

Shaheen Darani ${ }^{1 \star}$, Sandy Simpson ${ }^{1}$, Robert McMaster ${ }^{1}$, Elena Wolff ${ }^{2}$, Sarah Bonato ${ }^{3}$, Graham Glancy ${ }^{1}$ and Jason Quinn ${ }^{4}$

${ }^{1}$ University of Toronto and Centre for Addiction and Mental Health; ${ }^{2}$ University of Toronto, MD Program; ${ }^{3}$ Centre for Addiction and Mental Health and ${ }^{4}$ Department of Psychiatry, University of Western Ontario, London

${ }^{*}$ Corresponding author.

doi: 10.1192/bjo.2021.104

Aims. Mental illness amongst prison inmates is a prevailing issue across the world, as mental illnesses are overrepresented in correctional facilities when compared to community populations. Despite this, correctional officers receive little to no training on how to respond to inmates with mental illness. Implementing mental health training could improve officer knowledge, skills, and attitudes toward inmates with mental illness. This could lead to improvements in risk management, humane treatment of inmates, and interprofessional collaboration with healthcare providers. There is limited research on the educational value of inmate mental health training programs for correctional officers. As far as we are aware, there have been no prior reviews of this literature. The goal of the present study is to review this literature to explore the nature and effectiveness of correctional officer mental health training programs.

Method. Medical and criminal justice databases were searched for scientific articles describing correctional officer mental health training programs. All studies that included a measurable outcome on either correctional officer knowledge or inmate mental health were included in a final analysis. The review adhered to PRISMA guidelines for systematic reviews.

Result. Of 1492 articles identified using search terms, 11 were included in the analysis. 6 articles described mental health education programs, 2 articles described skill-specific programs, and 3 articles described suicide prevention programs. Training programs reviewed content about mental illness, practical skills, and included didactic and experiential teaching modalities. The programs led to improvements in knowledge, skills, and attitudes amongst officers. Prior mental health attitudes, knowledge, and work experience did not correlate with improvements following training. Officers were more receptive to program facilitators with correctional or lived mental health experience. Experiential teaching was preferred to didactic teaching. A decline in training improvements occurred several months after training.

Conclusion. There is limited but positive literature suggesting that structured training programs, particularly involving persons with lived experience and experiential components are beneficial. The decline in training improvements suggests need for ongoing education and systems change within correctional institutions to ensure sustainability of gains. In terms of limitations of this review, it is possible articles pertaining to correctional officer mental health training were not available on the databases searched or some programs may not be published. Studies were also limited in their outcome measurement, with no consistent tools, and no control groups. This review can guide the development, delivery, and contribute toward best practice guidelines for future inmate mental health training programs and studies.

\section{Exploring the views of university students with experience of common mental health disorders about support provided within primary care community settings}

\author{
Mikaela D’Arcy-Smith ${ }^{1 *}$ and Marta Buszewicz ${ }^{2}$ \\ ${ }^{1}$ UCL Medical School and ${ }^{2}$ Honorary Associate Professor - UCL \\ Department of Clinical, Educational and Health Psychology, \\ University College London \\ ${ }^{*}$ Corresponding author.
}

doi: 10.1192/bjo.2021.105

Aims. To assess the impact of common mental health disorders (CMHDs) on university students' function and wellbeing. To understand the barriers to receiving adequate support for CMHDs during both adolescence and at university. To provide feedback to healthcare professionals about how young people perceive the support provided when initially seeking help for psychological distress. To explore which forms of support students find the most useful.

Method. A literature review was initially undertaken, identifying the lack of prior research in this area. The current study addressed the gap by considering the needs of students with CMHDs in the context of primary care services, with a retrospective exploration of their views about support received during adolescence. 15 semi-structured qualitative interviews were conducted with both current university students and recent graduates from across the $\mathrm{UK}$, transcribed verbatim and subjected to thematic analysis. The study population included 7 men and 8 women, between the ages of 18-25 years.

Result. Five main themes emerged from the data:

The Journey to Disorder - Explored the difficulties faced by adolescents, and how these might contribute to their experience of CMHDs and their management.

Attitudes Towards Help-Seeking - Many participants had little trust in healthcare professionals as adolescents. This contributed to limited trust in university support systems as young adults.

Primary Care Support - Perceived effectiveness of General Practitioner (GP) support during adolescence in this cohort was highly variable. Although some participants described good experiences, others felt their views were ignored, with responsibility diverted to their caregivers. A lack of understanding from GPs about CMHDs in adolescents resulted in trust issues for them as young adults.

Recommendations for Change - Participants reflected on their previous and current experiences to inform suggestions for changes to tackle issue of psychological distress in adolescents.

Conclusion. Previous experiences of the care they had received when presenting with CMHDs during adolescence potentially affected the long-term wellbeing of university students and graduates; the initial support received was inconsistent with the needs of this age group. Recommendations for change included a greater emphasis on the importance of adolescent mental health education, tailoring interventions to personal growth and maturity, and ensuring primary healthcare providers are equipped with 
the skills required to manage psychological distress in young people.

\section{Mixed-methods service evaluation of a} multidisciplinary inpatient programme for functional neurological disorder and non-epileptic attack disorder

Peter Denno $^{1 \star}$, Samir Sholapurkar ${ }^{1}$, Elizabeth Mallam ${ }^{1}$ and Dane Rayment ${ }^{2}$

${ }^{1}$ North Bristol NHS Trust and ${ }^{2}$ North Bristol NHS

${ }^{\star}$ Corresponding author.

doi: 10.1192/bjo.2021.106

Aims. To evaluate a multidisciplinary inpatient treatment programme for Functional Neurological Disorder (FND) and Non-Epileptic Attack Disorder (NEAD), focussing on clinical effectiveness and patient experience. To produce recommendations for service development and future evaluation.

Method. We conducted a service evaluation of the multidisciplinary inpatient programme for FND and NEAD at the Rosa Burden Centre. We contacted all inpatients discharged between December 2019 and March 2020 via telephone in August/ September 2020. Quantitative outcomes were gathered on quality of life and psychological distress using the EQ-5D-5L and Core10 tools. Scores were compared to those gathered routinely at admission and discharge, using Wilcoxon's test for differences. Qualitative feedback on patient experience was gathered using open-ended prompts, and thematic analysis of this data was conducted independently by two researchers. Approval was gained from Southmead Clinical Audit Department (CE10237).

Result. 19 of 22 patients successfully completed the service evaluation. Quantitative results tended toward improvement on all measures between admission and discharge. Following discharge, there was a mixed pattern - sustained improvement in overall quality of life, but regression in other scores. Improvement in overall quality of life between admission and follow-up was statistically significant $(\mathrm{p}=0.012, \mathrm{Z}=2.52)$. Changes in psychological distress (Core10) were also statistically significant, reducing between admission and discharge $(\mathrm{p}=0.004, \mathrm{Z}=-2.84)$ and increasing between discharge and follow-up $(p=0.016, Z=2.42)$. Changes in other scores were not statistically significant at the $\mathrm{p}<0.05$ level. Qualitative results highlighted the value of the individual therapies offered, the multidisciplinary approach, and the supportive environment. Participants reported improved understanding of their diagnosis, and of self-management strategies. There was demand for greater access to psychological therapies, and increased provision of follow-up post-discharge. Some expressed dissatisfaction with the ward round format and excess "down-time". The programme was described as a "turning point” for 9 participants.

Conclusion. Quantitative results suggest the programme is associated with global improvement in quality of life, and postdischarge, some benefits are sustained while others are transient. However, interpretation is limited by sample size. We recommend further evaluation with a larger sample to replicate findings, assess effect sizes, and assess which patients or symptoms benefit most. To support this, we recommend improved collection of outcome measures, including routine collection of follow-up data. Positive qualitative findings highlight the strengths of the service and its value to patients. Recommendations for service development include recruiting a psychologist to provide further psychological therapy sessions; expanding the nurse-led follow-up service; and adjustments to the ward round format and activity programme.

\section{Listening to voices: understanding and} self-management of auditory verbal hallucinations in young adults

Peter Denno ${ }^{1 \star}$, Stephanie Wallis ${ }^{1}$, Kimberly Caldwell ${ }^{5}$, Jonathan Ives ${ }^{2}$, Stephen Wood ${ }^{3}$, Matthew Broome, Pavan Mallikarjun ${ }^{4}$, Femi Oyebode ${ }^{4}$ and Rachel Upthegrove ${ }^{4}$

${ }^{1}$ University of Birmingham, Medical School; ${ }^{2}$ University of Bristol; ${ }^{3}$ Orygen; ${ }^{4}$ University of Birmingham and ${ }^{5}$ Institute for Mental Health, School of Psychology, University of Birmingham ${ }^{\star}$ Corresponding author.

\section{doi: 10.1192/bjo.2021.107}

Aims. Auditory Verbal Hallucinations (AVH) are a hallmark of psychosis, but affect many other clinical populations. Patients' understanding and self-management of AVH may differ between diagnostic groups, change over time, and influence clinical outcomes.

We aimed to explore patients' understanding and selfmanagement of AVH in a young adult clinical population.

Method. 35 participants reporting frequent AVH were purposively sampled from a youth mental health service, to capture experiences across psychosis and non-psychosis diagnoses. Diary and photo-elicitation methodologies were used - participants were asked to complete diaries documenting experiences of $\mathrm{AVH}$, and to take photographs representing these experiences. In-depth, unstructured interviews were held, using participantproduced materials as a topic guide. Conventional content analysis was conducted, deriving results from the data in the form of themes.

Result. Three themes emerged:

(1) Searching for answers, forming identities - voice-hearers sought to explain their experiences, resulting in the construction of identities for voices, and descriptions of relationships with them. These identities were drawn from participants' life-stories (e.g., reflecting trauma), and belief-systems (e.g., reflecting supernatural beliefs, or mental illness). Some described this process as active / volitional. Participants described re-defining their own identities in relation to those constructed for AVH (e.g. as diseased, 'chosen', or persecuted), others considered AVH explicitly as aspects of, or changes in, their personality.

(2) Coping strategies and goals - patients' self-management strategies were diverse, reflecting the diverse negative experiences of AVH. Strategies were related to a smaller number of goals, e.g. distraction, soothing overwhelming emotions, 'realitychecking', and retaining agency.

(3) Outlook - participants formed an overall outlook reflecting their self-efficacy in managing AVH. Resignation and hopelessness in connection with disabling AVH are contrasted with outlooks of "acceptance" or integration, which were described as positive, ideal, or mature.

Conclusion. Trans-diagnostic commonalities in understanding and self-management of AVH are highlighted - answer-seeking and identity-formation processes; a diversity of coping strategies and goals; and striving to accept the symptom. Descriptions of "voices-as-self", and dysfunctional relationships with AVH, could represent specific features of voice-hearing in personality disorder, whereas certain supernatural/paranormal identities 